\title{
Association Between Antinuclear Antibodies and Interstitial Lung Diseases in Patients with Systemic Lupus Erythematosus
}

\author{
Osama S. DeifAllah, Walaa Elsayed Hamed, Mohamed Ali
}

Ismail.

Rheumatology \&amp; rehabilitation department, faculty of medicine, Sohag University, Sohag, Egypt

\begin{abstract}
:
Introduction: Systemic Lupus Erythematosus (SLE) is a disease primarily affecting women in their child-bearing years. SLE can affect any component of the respiratory system and manifest with pleuritis (with or without effusion), interstitial lung disease (ILD), alveolar hemorrhage, diaphragmatic weakness, pulmonary hypertension, airways disease, or pulmonary embolism. ILD occurs with a lower frequency in SLE compared with other connective tissue disease (CTD) subtypes. It is not known whether SLE-ILD differs from ILD in other CTD subtypes. Aim of the work: estimate the possible relationship between interstitial lung diseases and different antinuclear antibodies among patients with systemic lupus erythematosus.

Patients and Methods: Cross-sectional clinical study. A total of 100 SLE patients attending to Sohag University Hospitals will be included in the study. Patients included in this study will be classified as SLE patients according to either the 2012 SLICC criteria or the new 2017 ACR/EULAR SLE classification criteria. Results: ANA (antinuclear antibody) positivity, chest manifestations like (cough, dyspnea, expectoration, fever\&hemoptysis) are predictive variates for SLE-ILD. Conclusion: We observed a population of SLE-ILD and concluded that ANA (antinuclear antibody) positivity, chest manifestations like (cough, dyspnea, expectoration, fever\&hemoptysis) are predictive variates for SLE-ILD. Also, ANAIF speckled pattern has specificity to SLE-ILD and AntiSS-A/Ro52KD antibody is more specific to SLE-ILD. We recommend HRCT and PFT scans in these SLE patients.
\end{abstract}

Keywords: ANA, ILD, SLE

\section{Introduction}

Systemic Lupus Erythematosus (SLE) is a systemic inflame-mandatory autoimmune disease, primarily affecting women in their child-bearing years ${ }^{(1)}$.

SLE can affect any component of the respiratory system and manifest with pleuritis (with or without effusion), interstitial lung disease (ILD), alveolar hemorrhage, diaphragmatic weakness, pulmonary hypertension, airways disease, or pulmonary embolism ${ }^{(2)}$.

ILD occurs with a lower frequency in SLE compared with other connective tissue disease (CTD) subtypes. It is not known whether SLE-ILD differs from ILD in other CTD subtypes ${ }^{(3)}$.

The true prevalence of SLE-ILD is unknown, and estimates vary depending on the study design, ILD case definition, and study population. In one of the largest studies of biopsyproven CTD-associated ILD, among 93 subjects, Park and colleagues observed $30 \%$ had rheumatoid arthritis (RA), 38\% had systemic sclerosis (SSc), and only $1 \%$ had SLE ${ }^{(4)}$.

In a review of 90 SLE autopsies from 1958-2006, Quadrelli et al. noted that while $97.8 \%$ of patients had some form of pleuropulmonary involvement, the 
majority of parenchymal lesions were not attributable to SLE; strikingly, in their series, there was no case of acute or chronic SLEILD detected ${ }^{(5)}$.

Clinically apparent ILD is far less common in SLE than in other CTD, including RA, SSc, primary Sjogren's syndrome and poly/dermato-myositis. Across studies, the frequency of clinically significant ILD in SLE ranges from $1-15 \%{ }^{(6,7)}$.

There is no reliable serologic correlate for ILD in patients with SLE. Case series and cross-sectional studies have suggested a link between specific autoantibodies and ILD, specifically anti-Ro, anti-Sm, and anti-RNP ${ }^{(3)}$.

Findings from a study by Yee and colleagues challenge the relationship between autoantibodies and SLE-ILD: in a multivariate analysis of data from 348 subjects, increasing age and greater disease duration, but not autoantibodies, were associated with overall pulmonary damage ${ }^{(8)}$.

\section{Aim of the work:}

The aim of this study is to estimate the possible relationship between interstitial lung diseases and different antinuclear antibodies among patients with systemic lupus erythematosus.

\section{Patients and Methods:}

Design: an atelectatic clinical study. Patients:

A total of 100 SLE patients attending to Sohag University Hospitals will be included in the study.

Patients included in this study will be classified as SLE patients according to either the 2012 SLICC criteria or the new 2017 ACR/EULAR SLE classification criteria.

\section{Methods:}

- All of the participants will be subjected to the following:

○ Full history (demographic data and personal history, detailed history of general health condition and chronic or current diseases).

- General and systemic examination.

o Carful chest history and examination.

- Laboratory investigations including routine investigations (CBC, liver and renal functions).

○ PFT (pulmonary function tests).

o Imaging studies (Chest X-ray and high-resolution CT scan) for patients suspected to have chest problems.

o The abdominal US and Renal biopsy.

○ ANA detection by immunofluorescence and ANA profile using immunoblot method

\section{Results}

The mean age of our study group was 37 years+/- SD 12 years. $89 \%$ of our study group were females. $51 \%$ of our study group were from rural areas. Regarding SLE manifestations, $49 \%$ of our cases had a malar rash, only $1 \%$ had DLE, $76 \%$ had photosensitivity, $80 \%$ had oral or nasal ulcer, $8 \%$ had alopecia, 95\% had arthritis, 32\% had pleurisy, $5 \%$ had pericarditis, and only $4 \%$ had neurologic manifestations.

$87 \%$ of our cases had a cough, $33 \%$ had expectoration, $98 \%$ had dyspnea grade 2 to 3, 63\% had chest pain, 38\% had fever, and no one had hemoptysis. Mean ESR was 47, range from 10 to 145 , mean $\mathrm{P} / \mathrm{C}$ ratio (protein creatinine ratio) was 0.2 , range from 0.01 to 2.6 , mean ALT was 16, range from 3 to 73 . Mean AST was 17 and range from 4 to 62, and mean S.creatinine was 0.6, range from 0.3 to 1.9. Mean PLT (platelets) was 243, range from 17 to 596, mean MCV (mean corpuscular volume) was 78.9, range from 60 to 93, mean HB (hemoglobin) was 11.3, range from 7 to 14 and mean WBCs (white blood cells) was 6.8 and range from 1.2 to 17.3. 
Urine analysis of our cases shows urine pus was present in $1 \%$ only of our cases, the cast was granular in 3\% of cases and hyaline in $7 \%$ of them. Urine albumin was in $45 \%$ of cases, and sugar wasn't present in any case. Renal biopsy shows that $31 \%$ of our cases had class 2, $5 \%$ had class $2 \& 3,3 \%$ had class $3,3 \%$ had class 4 , and $58 \%$ of cases had no renal biopsy.

Abd U/S shows that $1 \%$ of our cases had a genetic kidney, $1 \%$ had hepatic hemangioma, $11 \%$ had hepatomegaly, $5 \%$ had mild ascites, $2 \%$ had nephropathy, $77 \%$ of cases were normal, and 3\% had splenomegaly. My chest x-ray $7 \%$ of our cases had cardiomegaly, $1 \%$ had exaggerated broncho vascular markings, $46 \%$ had non-homogenous opacities, $41 \%$ of our cases were normal, $3 \%$ had pericardial effusion, $2 \%$ had pleural effusion figure 1 .

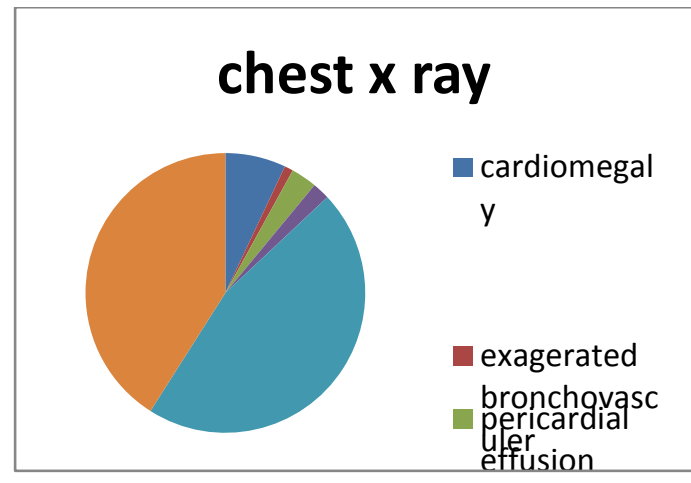

Figure 1. Chest x-ray

PFTs were normal in $67 \%$ of our cases and restrictive patterns in $33 \%$ of our cases.

By HRCT $1 \%$ of our cases had bilateral lower lung lobe atelectatic band \& pleural effusion, $34 \%$ had interstitial lung disease, $1 \%$ had left moderate pleural effusion\&partial lower lobe collapse, $45 \%$ of our cases were normal, $3 \%$ had pericardial effusion, $13 \%$ had pleural effusion, and $3 \%$ had pneumonitis.

ANAIF pattern was positive anticytoplasmic ribosomal antibody in $1 \%$ of our cases, positive homogenous in $24 \%$ of cases, $1 \%$ had homogenous\&speckled pattern, also $1 \%$ had a nucleolar pattern, and $68 \%$ had speckled pattern (Table 1).

\begin{tabular}{|c|c|c|}
\hline & $\begin{array}{l}\text { Numbe } \\
\mathrm{r}\end{array}$ & $\begin{array}{l}\text { Perce } \\
\text { nt }\end{array}$ \\
\hline Negative & 5 & $5 \%$ \\
\hline $\begin{array}{l}\text { Positive anticytoplasmic } \\
\text { ribosomal antibody }\end{array}$ & 1 & $1 \%$ \\
\hline $\begin{array}{l}\text { Positive homogenous \& } \\
\text { speckled }\end{array}$ & 1 & $1 \%$ \\
\hline Positive nucleoler & 1 & $1 \%$ \\
\hline Positive speckled & 68 & $68 \%$ \\
\hline Total & 100 & $100 \%$ \\
\hline
\end{tabular}

Table 1 shows the ANA pattern

Antinuclear antibodies percentage in our cases, the highest percentage were Anti SS-A/Ro60KD 44\%\&Anti dsDNA 41\% \&AntiSS-A/ Ro52KD 40 $\%$. Also other anti nuclear antibodies with smaller percentages as shown in table 2 .

\begin{tabular}{ccc}
\hline Antibody type & Number & Percent \\
\hline Nucleosome & 20 & $20 \%$ \\
DsDNA & 41 & $41 \%$ \\
Histones & 17 & $17 \%$ \\
Sm & 5 & $5 \%$ \\
RNP & 13 & $13 \%$ \\
Sm/RNP & 14 & $14 \%$ \\
SS-A/Ro60KD & 44 & $44 \%$ \\
SS-A/Ro52KD & 40 & $40 \%$ \\
SS-B/La & 8 & $8 \%$ \\
Scl70 & 4 & $4 \%$ \\
RNA-Polymerase & 1 & $1 \%$ \\
Ku & 2 & $2 \%$ \\
PM-Scl100 & 3 & $3 \%$ \\
Mi-2 & 2 & $2 \%$ \\
JO-1 & 3 & $3 \%$ \\
PCNA & 5 & $5 \%$ \\
Ribosome Po & 5 & $5 \%$ \\
DFS-70 & 4 & $4 \%$ \\
\hline
\end{tabular}

Table 2. ANA profile

We found that $44.1 \%$ of ILD cases had positive AntiSS-A/ Ro52KD, 38.2\% had positive AntiSS-A/ Ro60KD, also $38.2 \%$ had positive Anti dsDNA, $23.5 \%$ of ILD cases had positive AntiNucleosome, and $14.7 \%$ had positive Anti Histones. In our 34 cases with 
ILD, there were $70.59 \%$ of those cases having ANA speckled pattern, $20.59 \%$ of cases had a homogenous pattern, 2.94\% of cases had homogenous\&speckled pattern, 2.94 \%of cases had a nucleolar pattern, $2.94 \%$ of cases were negative.

In our 34 cases with ILD, there were $14.7 \%$ having ANAIF titer $1 / 40$, $29.4 \%$ of cases had titer $1 / 80,5.88 \%$ had titer $1 / 160,11.76 \%$ had titers $1 / 320,14.7 \%$ had titre $1 / 640,2.94 \%$ had titer $1 / 1520,8.82 \%$ had titer $1 / 2560,8.82 \%$ had titer $1 / 5120 \&$ $2.94 \%$ were negatives.

All cases with ILD had cough and dyspnea, only $85.3 \%$ had chest pain, $50 \%$ had a fever, $35.3 \%$ had expectoration\& no ILD cases had hemoptysis.

Regarding the relation between ANA different patterns\& chest manifestations in cases with ILD, cases with speckled pattern all of them had cough \&dyspnea, $37.5 \%$ had expectoration, $83.3 \%$ had chest pain, $50 \%$ had a fever and no one had hemoptysis. Cases with homogenous patterns all had cough, dyspnea and chest pain, $28.6 \%$ had expectoration, $71.4 \%$ had fever and no one had hemoptysis. Cases with homogenous \&speckled patterns all had cough, dyspnea and chest pain, no one had expectoration nor fever nor hemoptysis. Cases with nucleolar patterns all had a cough, dyspnea, chest pain but no expectoration, fever, hemoptysis. Cases with negative ANA IF had cough, dyspnea, expectoration but no chest pain, fever, hemoptysis.

When we made regression analysis between cases of ILD and those with no ILD, we found that ANA (antinuclear antibody) positivity, chest manifestations like (cough, dyspnea, expectoration, fever, and hemoptysis) are predictive variates for ILD as shown in table 3.

Multivariate regression analysis is done regarding ANA positivity and chest manifestations between ILD cases and cases with no ILD revealing that ANA and Chest manifestations were not risking factors together.

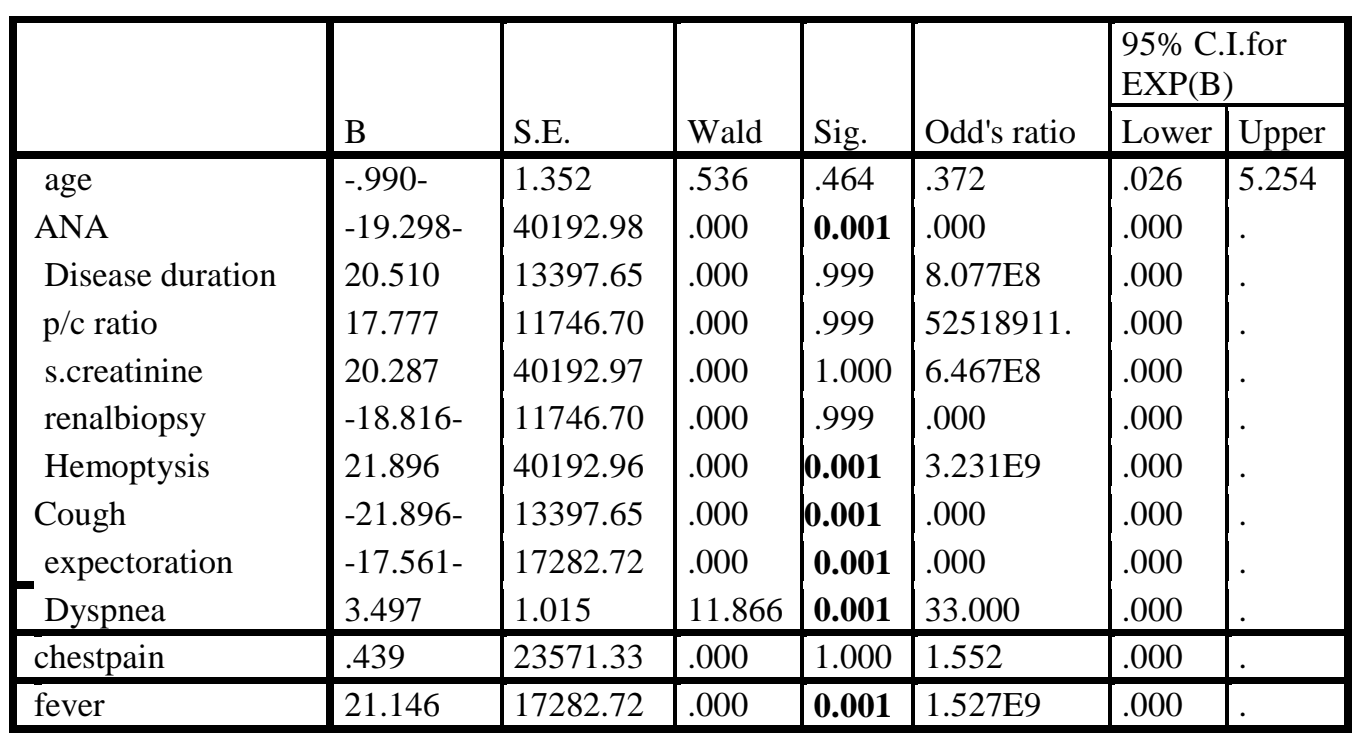

Table 3. Univariate Binary Regression Analysis between Cases of ILD and controls

\section{Discussion}

ILD can occur anytime during the course of SLE. On average, it develops in patients with disease duration of 10 years; rarely, it can be a presenting 
feature of SLE ${ }^{(9)}$. The frequency of SLE-related pulmonary damage increases with age and disease duration; thus, clinicians must have a heightened awareness for ILD as they follow SLE patients over time ${ }^{(6)}$. ILD symptoms are non-specific, variable among patients, often insidious, and do not necessarily correspond with radiographic or histologic evidence of ILD. Moreover, less than a third of patients with radiographic evidence of ILD are symptomatic (10). When symptoms arise, dyspnea on exertion, a chronic nonproductive cough, and pleuritic chest pain are common ${ }^{(10)}$.

Previous studies suggested that 2-8\% of ILD occurred in SLE and most patients with multisystem disease ${ }^{(11)}$. According to the report of Ghosh A, et al. from India, the prevalence of SLEILD was about $10 \%{ }^{(12)}$. While Fenlon et al. ${ }^{(13)}$ declared that ILD could be found in one-third of the SLE patients. In a case-series of 18 SLE patients with radiographic evidence of ILD, all had exertional dyspnea, 69\% had a productive cough, $66 \%$ had pleuritis, $44 \%$ had unilateral diaphragm elevation, and $66 \%$ had basilar crackles. Cyanosis and clubbing are rare ${ }^{(14)}$. Abrupt onset of fever, cough, pleuritic chest pain and tachypnea commonly mark acute lupus pneumonitis or DAH, a presentation often seen in younger patients (mean age 38 years) and associated with a mortality rate of greater than $50 \%{ }^{(15)}$.

HRCT is a sensitive and reasonably accurate tool for the detection of ILD. The previous histological reports about ILD diagnosed by HRCT in SLE were mostly in non-specific interstitial pneumonia (NSIP) pattern ${ }^{(16)}$, though usual interstitial pneumonia (UIP), lymphocytic interstitial pneumonia (LIP), and organizing pneumonia (OP) all have been described ${ }^{(16)}$.

By HRCT $1 \%$ of our cases had bilateral lower lung lobe atelectatic band\&pleural effusion, $34 \%$ had interstitial lung disease, $1 \%$ had left moderate pleural effusion\& partial lower lobe collapse, $45 \%$ of our cases were normal, $3 \%$ had pericardial effusion, $13 \%$ had pleural effusion, and $3 \%$ had pneumonitis.

It is previously reported that histological analysis could be suggested by HRCT characteristics, such as honeycombing, basilar predominant reticulation and traction bronchiectasis with limited ground glass opacities for usual interstitial pneumonia (UIP), as well as predominant bilateral basilar groundglass opacities with small reticulation and absence of honeycombing for nonspecific interstitial pneumonia (NSIP) (17). ILD patients with positive antiSSA may be described as a homogeneous group with more ground-glass opacity and more severe lung function impairment than those with ILD with negative anti-SSA antibody ${ }^{(17)}$.

ANAIF pattern was positive anticytoplasmic ribosomal antibody in $1 \%$ of our cases, positive homogenous in $24 \%$ of cases, $1 \%$ had homogenous\&speckled pattern, also $1 \%$ had a nucleolar pattern, and $68 \%$ had speckled pattern. Antinuclear antibodies percentage in our cases, the highest percentage were Anti RO60 44\% \& Anti dsDNA 41\% \&Anti RO52 $40 \%$. Also other antinuclear antibodies with smaller percentages.

$44.1 \%$ of ILD cases in this study had positive Anti RO52, 38.2\% had positive Anti RO60, also $38.2 \%$ had positive Anti dsDNA, $23.5 \%$ of ILD cases had positive Anti nucleosome, and $14.7 \%$ had positive Anti histone.

In our 34 cases with ILD there were $70.59 \%$ of those cases having ANA speckled pattern, $20.59 \%$ of cases had the homogenous pattern, $2.94 \%$ of cases had homogenous\&speckled pattern, $2.94 \%$ of cases had nucleolar 
pattern, $2.94 \%$ of cases were negative. Additionally, in our 34 cases with ILD there were $14.7 \%$ having ANA IF titer $1 / 40,29.4 \%$ of cases had titer $1 / 80$, $5.88 \%$ had titre $1 / 160,11.76 \%$ had titer $1 / 320,14.7 \%$ had titre $1 / 640,2.94 \%$ had titer $1 / 1520,8.82 \%$ had titer $1 / 2560,8.82 \%$ had titer $1 / 5120 \&$ $2.94 \%$ were negative.

The relation between ANA different patterns\& chest manifestations in our cases with ILD, cases with speckled pattern all of them had cough and dyspnea, $37.5 \%$ had expectoration, $83.3 \%$ had chest pain, $50 \%$ had fever\& no one had hemoptysis. Cases with homogenous patterns all had cough, dyspnea, chest pain, $28.6 \%$ had expectoration, $71.4 \%$ had fever and no one had hemoptysis. Cases with homogenous \&speckled patterns all had cough, dyspnea, chest pain, no one had expectoration nor fever nor hemoptysis. Cases with nucleolar patterns all had a cough, dyspnea, chest pain but no expectoration, fever, hemoptysis. Cases with negative ANA IF had cough, dyspnea, expectoration but no chest pain, fever, hemoptysis.

According to the previously described relationship between anti-SSA/Ro60 and $\operatorname{SLE}{ }^{(18,19)}$, this anti-Ro specificity was found to be positively associated with hypocomplementemia, an SLE related immunological feature. On the other hand, a negative association was observed between anti-Ro52/TRIM21 and anti-dsDNA which is consistent with the higher prevalence of this antibody in other autoimmune conditions such as SS, myositis, SSc, and liver diseases ${ }^{(20,21)}$.

When we made regression analysis between cases of ILD and those with no ILD, we found that ANA (antinuclear antibody) positivity, chest manifestations like (cough, dyspnea, expectoration, fever, and hemoptysis) are predictive variates for ILD. Multivariate regression analysis is done regarding ANA positivity and chest manifestations between ILD cases and cases with no ILD revealing that ANA and Chest manifestations were not risking factors together.

Similar to our study, when Lian et al. (22) done univariate logistic regression was performed to evaluate all clinical and laboratory parameters. Among all the factors, positive SSA, positive SSB, positive ACL, and positive anti-U1RNP were significantly associated with the occurrence of interstitial lung disease in SLE. In contrast to ours, in their study, when variables associated with outcome at $\mathrm{P}<0.20$ were then summarized into the multivariate logistic model for the calculation of adjusted odds ratio. Positive SSA, positive SSB, positive anti-Scl-70 and dry eyes/mouth turned out to be significant, which could be considered primarily the predictors for interstitial lung disease in SLE.

\section{Conclusion:}

We observed a population of SLEILD and concluded that ANA (antinuclear antibody) positivity, chest manifestations like (cough, dyspnea, expectoration, fever\&hemoptysis) are predictive variates for SLE-ILD. Also, ANAIF speckled pattern has specificity to SLE-ILD and AntiSS-A/Ro52KD antibody is more specific to SLE-ILD. We recommend HRCT and PFT scan in these SLE patients. As most ILD lesions are sensitively observed in HRCT, it is not absolutely applied lung biopsy. Considering that the present study is a retrospective clinical observation and the samples are relatively small, further biological research and prospective study with lager data are required to confirm the predictive value of the indices. 


\section{References:}

1. Chakravarty EF, Bush TM, Manzi S, Clarke AE, Ward MM. Prevalence of adult systemic lupus erythematosus in California and Pennsylvania in 2000: estimates obtained using hospitalization data. Arthritis Rheum. 2007;56(6):2092-4.

2. Pines A, Kaplinsky N, Olchovsky D, Rozenman J, Frankl O. Pleuropulmonary manifestations of systemic lupus erythematosus: clinical features of its subgroups. Prognostic and therapeutic implications. Chest. 1985;88(1):129-35.

3. Mittoo S, Fischer A, Strand V, Meehan R, Swigris JJ. Systemic Lupus Erythematosus-Related Interstitial Lung Disease. Current Rheumatology Reviews. 2010;6:99-107.

4. Park JH, Kim DS, Park IN, Jang SJ, Kitaichi M, Nicholson AG, et al. Prognosis of fibrotic interstitial pneumonia: idiopathic versus collagen vascular disease-related subtypes. American journal of respiratory and critical care medicine. 2007;175(7):705-11.

5. Quadrelli SA, Alvarez C, Arce SC, Paz L, Sarano J, Sobrino EM, et al. Pulmonary involvement of systemic lupus erythematosus: analysis of $q$. necropsies. Lupus. 2009;18(12):105360.

6. Bertoli AM, Vila LM, Apte M, Fessler BJ, Bastian HM, Reveille JD, et al. Systemic lupus erythematosus in a multiethnic US Cohort LUMINA XLVIII: factors predictive of pulmonary damage. Lupus. 2007;16(6):410-7.

7. Lee SS, Singh S, Link K, Petri M. High-sensitivity C-reactive protein as an associate of clinical subsets and organ damage in systemic lupus erythematosus. Seminars in arthritis and rheumatism. 2008;38(1):41-54.

8. Yee CS, Hussein H, Skan J, Bowman $\mathrm{S}$ 'Situnayake D, Gordon C. Association of damage with autoantibody profile, age, race, sex and disease duration in systemic lupus erythematosus.

Rheumatology. 2003;42(2):276-9.

9. Takada H, Saito Y, Nomura A, Ohga S, Kuwano K, Nakashima $\mathrm{N}$, et al. Bronchiolitis obliterans organizing pneumonia as an initial manifestation in systemic lupus erythematosus. Pediatric pulmonology. 2005;40(3):257-60.

10. Swigris JJ, Fischer A, Gillis J, Meehan RT, Brown KK. Pulmonary and thrombotic manifestations of systemic lupus erythematosus. Chest. 2008;133(1):271-80.

11.Pego-Reigosa JM, Medeiros DA, Isenberg DA. Respiratory manifestations of systemic lupus erythematosus: old and new concepts. Best practice \& research Clinical rheumatology. 2009;23(4):469-80.

12. Ghosh A, Das T, Ghosh A, Karmakar $\mathrm{P}, \mathrm{Pal}$ J. Evaluation of respiratory manifestations in systemic lupus erythematosus with special reference to pulmonary interstitial involvement. Journal of the Indian Medical Association. 2012;110(2):109-11.

13.Fenlon HM, Doran M, Sant SM, Breatnach E. High-resolution chest CT in systemic lupus erythematosus. AJR American journal of roentgenology. 1996;166(2):301-7.

14.Eisenberg H, Dubois EL, Sherwin RP, Balchum OJ. Diffuse interstitial lung disease in systemic lupus erythematosus .Annals of internal medicine. 1973;79(1):37-45.

15.Keane MP, Lynch JP, 3rd. Pleuropulmonary manifestations of systemic lupus erythematosus. Thorax. 2000;55(2):159-66.

16. Devaraj A, Wells AU, Hansell DM. Computed tomographic imaging in connective tissue diseases. Seminars in respiratory and critical care medicine. 2007;28(4):389-97.

17.Boitiaux JF, Debray MP, NicaiseRoland P, Adle-Biassette H, Danel C, Clerici $\mathrm{C}$, et al. Idiopathic interstitial lung disease with the anti-SSA antibody. Rheumatology. 2011;50(1.0.- - Y Y $\leqslant 0:(Y$

18. Menendez A, Gomez J, Escanlar E, Caminal-Montero L, Mozo L. Clinical associations of anti-SSA/Ro60 and 
anti-Ro52/TRIM21 antibodies:

Diagnostic utility of their separate detection. Autoimmunity. 2013;46(1):32-9.

19.Dugar M, Cox S, Limaye V ،Gordon TP, Roberts-Thomson PJ. Diagnostic utility of anti-Ro52 detection in systemic autoimmunity. Postgraduate medical journal. 2010;86(1012):79-82.

20.Brouwer R, Hengstman GJ, Vree Egberts W, Ehrfeld H, Bozic B, Ghirardello A, et al. Autoantibody profiles in the sera of European patients with myositis. Annals of the rheumatic diseases. 2001;60(2):116-23.

21.Schulte-Pelkum J, Fritzler M, Mahler M. Latest update on the Ro/SS-A autoantibody system. Autoimmunity reviews. 2009;8(7):632-7.

22. Lian F, Jun Zhou, Yu Wang, Wei Cui, Dongying Chen, Clinical features and independent predictors of interstitial lung disease in systemic lupus erythematosus, Int $\mathrm{J}$ Clin Exp Med 2016;9(2):4233-4242. 